\title{
Quantum transport in carbon nanotubes
}

\author{
M. Baxendale*, M. Melli ${ }^{\dagger, *}$ and I. E. Pollini ${ }^{\dagger}$ \\ * Department of Physics, Queen Mary, University of London, London, UK \\ ${ }^{\dagger}$ Dipartimento di Fisica, Università degli Studi Milano, 20133 Milano, Italy
}

\begin{abstract}
In the last twenty years, due to technological advances, it has become possible to build, manipulate and observe nanometer structures and so their dimensions are intermediate between the microscopic and macroscopic. These new systems present novel features and have originated the new field of mesoscopic physics As for the transport properties the mesoscopic conductors do not have ohmic behaviour and the free mean path of electrons is longer than the conductor length in some systems. In this case the conductor is called ballistic, $i$. e. the electron can travel without scattering in the bulk of the conductor. Another significant consequence of the conductor low dimensionality is that its conductance $G=I / V$ is quantized. The current approach to describe these phenomena is the transmission formalism introduced by Landauer: the current through a conductor is given by the probability that an electron can transmit through it. [1]. Carbon nanotubes (CNT), which are cylindrical macromolecules formed by periodic hexagonal structures of carbon atoms and typically 1 to $5 \mathrm{~nm}$ wide and 1 to $10 \mu \mathrm{m}$ long, should be in ideal systems to proof the theories about the mesoscopic transport.The nature of the electronic transport in CNT is intriguing and still a matter of debate, and only a few experiments have shown the conductance quantization in CNT [2, 3]. In this work we present new transport data on carbon double wall nanotubes (DWNT) by means of the mechanically controllable break junction (MCBJ) technique [4]. We assume that scattering in the bulk is negligible and the number of channels determines the average conductance in the ballistic regime. A major conclusion of our work is the realization that actually there are two types of ballistic pathways in carbon nanotubes networks. Further theoretical and experimental studies at high voltages seems necessary to better understand the DWNT properties.
\end{abstract}

\section{REFERENCES}

1. R. Landauer, IBM J. Res. Dev. 1, 223 (1957)

2. S. Frank et al.,"Carbon nanotubes quantum resistors", Science 280, 1744 (1998)

3. A. Urbina et al., "Quantum conductance steps in solutions of multiwalled carbon nanotubes", Phys. Rev. Lett. 90, 106603 (2003)

4. K. Hansen et al.,"Quantized conductance in relays“, Phys. Rev. B 56, 2208 (1997) 